\title{
Research on Robust Optimization in Airlines' Hybrid Channel Coordination Considering Service Level
}

\author{
Lijun Zheng ${ }^{*}$, Rong Hu and Feifei Zhang \\ No.29 Jiangjun Avenue, Jiangning District, Nanjing, Jiangsu Province, China \\ ${ }^{*}$ Corresponding author
}

Keywords: Service level, Channel coordination, Robust optimization, Uncertainty.

\begin{abstract}
This paper considers service level in the study of airlines' hybrid channel coordination, which has great impact on airlines' operation and performance. Firstly, the service level was expressed by demand function and a robust optimization model of airlines' hybrid channel coordination with uncertain demands was established based on the channel coordination features and scenario analysis. Then numerical experiments of the robust optimization model were conducted to explore the relationship between service level, distribution channel ratio, profits and system robustness. The results show that the model is robust to uncertain market needs; airlines should pay attention to the influence of service level on themselves and maintain the optimal service level, which can not only improve the system robustness but also increase their own revenue; agents should seize opportunities, focus on reducing channel costs, and develop cooperation to improve service level to attract passengers; airlines and their agents should work together to achieve a win-win situation.
\end{abstract}

\section{Introduction}

In recent years, the traffic volume of China's civil aviation market has grown rapidly with an average annual growth rate of over $12 \%$, which has brought more travel choices and conveniences to passengers. However, some problems of airlines' hybrid channels still exist, such as illegal ticket agents, which not only infringes passengers' rights and interests, but also damage airlines' image and interests. With the continuous advancement of China's civil aviation power, airlines put forward higher requirements for marketing management level and service level, and the development of airlines' hybrid channels is bound to usher in a new stage.

The research on optimization and management of airlines' hybrid channel coordination has achieved fruitful achievement. Jiang [1] studied the uncertainty of market supply and demand, operation costs and agreement adjustment to analyzed the impact of these factors. Cheng [2] took American airlines as research objects and explored the coordination of network direct channels and traditional channels under the conditions of online market. Zhang and Ding used evolutionary game theory to explore the strategic choice of airlines' hybrid channels [3]. Saha [4] and David [5] studied the dual channel coordination from the aspects of channel competition and channel structure. Regarding the uncertain parameters, the existing literature commonly uses scenario analysis method and interval analysis method. Based on channel coordination characteristics, Jiang and Hu [6-7] took scenario analysis to establish a robust optimization model of airlines' hybrid channel coordination with uncertain demands. Then, they inputted numerical values for robust model simulation, by which verified the robustness of the model and the results.

Concerning the qualitative theory of airlines' service level, Xie[8] analyzed the significance of service quality, summed up the relationship between customer satisfaction, customer stickiness and airline efficiency and emphasized the importance of service level. Yu [9] described the huge impact of service quality differences on customer perception through specific cases, emphasizing the importance of service level to customer experience and airlines. From qualitative to empirical research, Ma [10] discussed various problems that will arise in the process of civil aviation service, and has certain reference function for the improvement of service quality. Zhao [11] combined service quality theory with specific cases and proposed two points: first, airlines should analyze 
products and services that the passengers really need through actual data; second, airlines should improve service level and formulate corresponding system specifications. From the management point of view, $\mathrm{Ni}$ [12] formulated a set of specifications for effectively selecting high-quality service personnel on aspects of training, quality and assessment, which gave airlines a qualitative reference.

At present, many beneficial research results have been obtained in the areas of airlines' hybrid channel coordination and qualitative research of service level. However, the above research mainly focuses on qualitative issues of civil aviation operation, the study combined the airlines' hybrid channel coordination with service level is still lacking.

In light of the above discussion, we make the following specific contributions. First, this paper takes the impact of service level into consideration and uses demand function to represent the service level. Second, this paper establishes a robust optimization model of airlines' hybrid channel coordination by scenario analysis and deeply investigates the relationship between service level, coordination system profits and system robustness through numerical experiments.

The remainder of this paper is structured as follows: Section 2 introduces the airlines' hybrid channel coordination model with service level and uncertain demands; Section 3 conducts numerical experiments to verify the model robustness using scenario analysis and analyze the specific impact of service level; Section 4 draws the research conclusions and suggestions for future study.

\section{Modeling}

\section{Airlines’ Hybrid Channel Coordination System}

The system of airlines' hybrid channel coordination includes airlines, agents and passengers (as shown in Fig.1). Passengers buy different kinds of air tickets from airlines or agents while airlines sell tickets to passengers with uncertain demands directly or through different agents. The objective of this system is to maximize the total profits of all airlines and agents.

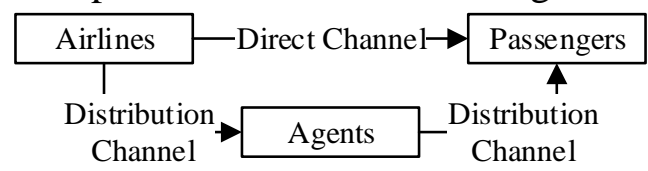

Figure 1. Airlines’ hybrid channel coordination system[13]

Concerning the current market environment, homogenized civil aviation services can no longer meet the individualized and differentiated consumer demand. In order to cope with such problems, the study of the optimal service level has emerged. As we all know, there is a positive relationship between service level, operation costs, that is, the higher the service level, the higher the operation costs. Under current conditions, airlines are faced with such difficulties. On the one hand, they must upgrade their service level in order to maintain passenger relations, but this will inevitably lead to a rise in service costs. On the other hand, airlines need to reduce costs, which will lead to a decline in service quality, resulting in reduced profits. Therefore, in order to balance such contradictions, the decision of the optimal service level is crucial which can not only provide good services to passengers but also avoid excessive cost loss.

\section{Notations}

We use the subscripts $i, j, k, l$ to express the air tickets, airlines, passengers and agents separately. Other parameters and decision variables and their definitions are as follows.

Parameters:

$e_{i j k}$, unit profit of airline $j$ by selling air ticket $i$ to passenger $k$;

$e_{i l k}$, unit profit of agent $l$ by selling air ticket $i$ to passenger $k$;

$c_{i j k}$, unit variable cost of airline $j$ by selling air ticket $i$ to passenger $k$;

$C_{i j}$, unit variable cost of airline $j$ to cope with agent $l$; 
$c_{i l k}$, unit variable cost of agent $l$ to sell air ticket $i$ to passenger $k$;

$r_{i j}$, preparation cost of airline $j$ to produce air ticket $i$;

$f_{j k}$, fixed cost of airline $j$ to satisfy demands of passenger $k$;

$f_{l j}$, fixed cost of airline $j$ to cope with agent $l$;

$f_{i k}$, fixed cost of agent $l$ to cope with passenger $k$;

$C_{i j}$, maximum transportation capacity of air ticket $i$ of airline $j$;

$T_{j}$, total transportation capacity of airline $j$;

$d_{i k}$, demands of air ticket $i$ from passenger $k$;

$q_{i k}$, unit penalty cost if the quantity of air ticket $i$ does not meet the demands of passenger $k$;

$t$, the lower limit of the distribution channel ratio, $t \in(0,1]$;

$\alpha$, service level of airlines;

$\alpha^{*}$, the optimal service level of airlines;

$s$, the number of possible scenarios of the passengers' uncertain demands, $G_{s}=\{1,2, \mathrm{~L} s\}$;

$m$, impact of service level $\alpha$ on demand $(m>0)$;

$n$, constant;

Decision variables:

$x_{i j k}$, quantity of air ticket $i$ that passenger $k$ purchases from airline $j$;

$x_{l i k}$, quantity of air ticket $i$ that passenger $k$ purchases from agent $l$;

$z_{j k} \in\{0,1\}$, if airline $j$ meets the demands of passenger $k$, then $z_{j k}=1$, otherwise $z_{j k}=0$;

$u_{i k}$, quantity of air ticket $i$ that does not meet the demands of passenger $k$;

$y_{i j} \in\{0,1\}$, if airline $j$ offers air ticket $i$, then $y_{i j}=1$, otherwise $y_{i j}=0$.

\section{Optimization Model}

Based on the cost and profits structure of airlines' hybrid channel coordination system, the objective function is established with the objective of the maximum total profits including both airlines and agents:

$$
\begin{aligned}
& \operatorname{maxP}=\sum_{s \in G_{s}} p_{s}\left[\sum_{i} \sum_{j} \sum_{k} e_{i j k} x_{i j k}^{s}+\sum_{l} \sum_{i} \sum_{k} e_{i l k} x_{i l k}^{s}-\sum_{i} \sum_{j} \sum_{k} c_{i j k} x_{i j k}^{s}-\sum_{l} \sum_{i} \sum_{j} c_{i j j} x_{i l k}^{s}-\right. \\
& \left.\sum_{i} \sum_{j} r_{i j} y_{i j}-\sum_{j} \sum_{k} f_{j k} z_{j k}^{s}-\sum_{l} \sum_{j} f_{l j}-\sum_{l} \sum_{k} f_{l k}-\sum_{l} \sum_{i} \sum_{k} c_{i k} x_{i l k}^{s}-\sum_{i} \sum_{k} q_{i k} u_{i k}^{s}\right]
\end{aligned}
$$

Where, $\sum_{j} \sum_{i} \sum_{k} e_{i j k} x_{i j k}^{s}$ represents airlines' total profits under scenario $s ; \sum_{l} \sum_{i} \sum_{k} e_{i k} x_{i k k}^{s}$ represents agents' total profits under scenario $s ; \sum_{i} \sum_{j} \sum_{k} c_{i j k} x_{i j k}{ }^{s}$ represents airlines' total variable costs under scenario $s ; \sum_{i} \sum_{j} \sum_{l} c_{i j l} x_{i k l}{ }^{s}$ represents agents' total variable costs paid by airlines under scenario $s$; $\sum_{i} \sum_{j} r y_{i j}$ represents airlines' total production preparation costs; $\sum_{j} \sum_{k} f_{j k} z_{j k}{ }^{s}$ represents airlines' total fixed costs under scenario $s ; \sum_{j} \sum_{l} f_{j l}$ represents agents' total fixed costs paid by airlines; $\sum_{k} \sum_{l} f_{k l}$ represents agents' total fixed costs; $\sum_{i} \sum_{k} \sum_{l} c_{i k l} x_{i k l}{ }^{s}$ represents agents' total variable costs under scenario $s ; \sum_{i} \sum_{k} q_{i k} u_{i k}{ }^{s}$ represents total penalty costs of unsatisfied demands under scenario $s$.

Considering the high homogeneity of air tickets sold by airlines and agents, it is assumed that demand is correlated with service level positively and only influenced by it. According to the demand 
function proposed by Dye [14], this paper constructs the function of demand $d_{i k}^{s}$ about service level $\alpha$ as follows:

$$
d_{i k}^{s}=m \alpha+n, \forall s, i, k
$$

The service level in this paper represents the degree of satisfaction with market demand:

$$
\alpha=\frac{\sum_{j} x_{i j k}{ }^{s}}{d_{i k}{ }^{s}}, \forall s, i, k
$$

Thus, the formula for solving the optimal service level $\alpha^{*}$ can be derived.

$$
\frac{\sum_{j}{x_{i j k}{ }^{s}}_{\alpha}}{\alpha}=m \alpha+n, \forall s, i, k
$$

Other specific constraints are as follows:

Total transportation capacity constraint of air ticket:

$$
\sum_{k} X_{i j k}^{s}+\sum_{l} X_{i l k}^{s}-y_{i j} C_{i j} \leq 0, \forall s, i, j
$$

Total transportation capacity constraint of airlines:

$$
\sum_{i} \sum_{k} x_{i j k}^{s}+\sum_{l} \sum_{i} \sum_{k} x_{i l k}^{s} \leq T_{j}, \forall s, j
$$

The lower limit of the distribution channel ratio:

$$
\sum_{i} \sum_{k} \sum_{l} x_{i k l} \geq \mathrm{t}\left(\sum_{i} \sum_{k} x_{i j k}+\sum_{i} \sum_{k} \sum_{l} x_{i k l}\right), \forall j
$$

The balance constraint between supply and demand:

$$
\sum_{j} x_{i j k}+\sum_{l} x_{i k l}+u_{i k}=d_{i k}, \forall i, k
$$

The airlines’ supply does not exceed the total passenger demand:

$$
x_{i j k}^{s} \leq \sum_{k} d_{i k}^{s}, \forall s, i, j
$$

The agents' supply does not exceed the total passenger demand:

$$
x_{l i k}^{s} \leq \sum_{k} d_{i k}^{s}, \forall s, i, l
$$

Non-negative constraints:

$$
\begin{aligned}
& x_{i j k} \geq 0, \quad x_{i k l} \geq 0, \quad u_{i k} \geq 0, \quad z_{j k} \in\{0,1\}, \\
& y_{i j} \in\{0,1\}, \quad \forall i, j, k, l
\end{aligned}
$$

\section{Numerical Experiments}

Based on the relevant data of the literature [13], we conduct comparative analysis with two sets of data of certain demand and uncertain demand for to verify the robustness of the model and its solution, and analyze the specific impact of service level on several aspects.

\section{Robustness Verification}

In the hybrid channel coordination system, the demand on different tickets for each passenger is uncertain. This paper assumes four demand scenarios, the probability is shown in Table 1. In addition, we assume that the preparation cost of airlines is $51 \mathrm{RMB}$; the fixed cost of agents paid by airlines is 
$25 \mathrm{RMB}$, the airline's maximum capacity is 3000 seats; the unit variable cost is 5,3 and $2 \mathrm{RMB}$; the lower limit of the agent ratio is $60 \%$, and the values of other parameters are listed in Table 2 to Table 4.

Table 1. Demand scenarios of passengers (unit: seat)

\begin{tabular}{|c|c|c|c|c|c|c|}
\hline \multicolumn{2}{|l|}{ Scenario } & $\begin{array}{l}\text { Probabilit } \\
\mathrm{y}\end{array}$ & $\begin{array}{l}\text { Busines } \\
\mathrm{s}\end{array}$ & $\begin{array}{l}\text { Leisur } \\
\mathrm{e}\end{array}$ & Visiting relatives & $\begin{array}{l}\text { Other } \\
\mathrm{s}\end{array}$ \\
\hline & 1 & 0.1 & 177 & 160 & 159 & 148 \\
\hline Uncertain & 2 & 0.2 & 179 & 196 & 183 & 145 \\
\hline demand & 3 & 0.3 & 385 & 356 & 325 & 240 \\
\hline & 4 & 0.4 & 560 & 480 & 435 & 368 \\
\hline Certain dema & and & 1 & 393 & 354 & 324 & 263 \\
\hline
\end{tabular}

Table 2. Unit variable cost of passengers and per unit penalty of unsatisfied demands (unit: RMB)

\begin{tabular}{lllll}
\hline & Busines & Leisur & Visiting relatives & $\begin{array}{l}\text { Other } \\
\text { s }\end{array}$ \\
\hline Sariable cost & 19 & 11 & 17 & 21 \\
Penalty & 59 & 31 & 30 & 41 \\
\hline
\end{tabular}

Table 3. Unit variable cost of agents to cope with passengers (unit: RMB)

\begin{tabular}{lllll}
\hline $\begin{array}{l}\text { Agen } \\
\mathrm{t}\end{array}$ & $\begin{array}{l}\text { Busines } \\
\mathrm{s}\end{array}$ & $\begin{array}{l}\text { Leisur } \\
\mathrm{e}\end{array}$ & Visiting relatives & $\begin{array}{l}\text { Other } \\
\mathrm{s}\end{array}$ \\
\hline 1 & 27 & 34 & 25 & 40 \\
2 & 28 & 31 & 25 & 36 \\
3 & 21 & 35 & 37 & 29 \\
\hline
\end{tabular}

Table 4. Fixed cost of airlines and agents to meet passengers' demands (unit: RMB)

\begin{tabular}{lllll}
\hline & $\begin{array}{c}\text { Bus } \\
\text { iness }\end{array}$ & $\begin{array}{l}\text { Leisur } \\
\text { e }\end{array}$ & Visiting relatives & $\begin{array}{l}\text { Other } \\
\text { s }\end{array}$ \\
\hline Airline & 39 & 31 & 26 & 19 \\
Agent 1 & 34 & 26 & 15 & 16 \\
Agent 2 & 46 & 34 & 21 & 33 \\
Agent 3 & 45 & 35 & 20 & 27 \\
\hline
\end{tabular}

The robustness represents the stability of a system. When there are uncertainties inside and outside the system, the smaller the deviation rate presented by the system, the stronger the robustness of the system. Through simulation by LINGO, the maximum total profits are calculated to be $27815 \mathrm{RMB}$ with certain demand and 26583 RMB with uncertain demand, and the optimal service level is 0.8106. The total profits deviation rate of the airline's hybrid channel coordination system is $4.43 \%$, which means the difference of the optimal solution with certain and uncertain demand is small, thus, the model has high robustness.

\section{The Impact of Distribution Channel Proportion on the Optimal Service Level}

Concerning the airlines' hybrid channels, the current trend is that airlines are taking various measures to increase their direct channel ratio, which means airlines encourage passengers to buy tickets through direct channel and results in a decrease of distribution channel ratio. Therefore, the ratio of agents in this paper shows a downward trend.

We keep the values of other parameters in Section 3.1 unchanged and set the distribution channel ratio as $\mathrm{t}=\{0.9,0.8,0.7,0.6,0.5,0.4,0.3\}$ for the numerical simulation to observe the change of the optimal solution of the model. The numerical simulation results are shown in Table 5. 
Table 5. The optimal solutions under different distribution channel ratios

\begin{tabular}{lll}
\hline $\mathrm{t}$ & $\alpha^{*}$ & $\mathrm{P}(\mathrm{RMB})$ \\
\hline 0.9 & 0.7357 & 15735 \\
0.8 & 0.7634 & 18685 \\
0.7 & 0.7957 & 21375 \\
0.6 & 0.8106 & 27935 \\
0.5 & 0.8424 & 28905 \\
0.4 & 0.9033 & 32425 \\
0.3 & 0.9374 & 36465 \\
\hline
\end{tabular}

As shown in Table 5, with the decrease of the distribution channel ratio $t$, the airline's optimal service level $\alpha^{*}$ continues to increase. This is due to the increase of airline's direct channel ratio, which improves passengers' loyalty. In this case, airlines should seize the opportunity to cultivate a fixed customer group and maximize the satisfaction of passengers' needs, so the optimal service level can be improved continuously. In addition, airlines also need to consolidate passengers' preference for airlines, thereby further increasing the ratio of direct channels, thus forming a benign circulatory system.

At the same time, the total profits of the system also show a growing trend which implies the necessity for airlines to improve the direct channel ratio. This will not only improve airlines' brand benefit, but also bring more profits for the hybrid channel system.

\section{The Impact of Service Level on Profits}

With the rapid development of the civil aviation market, passengers have higher and higher requirements for service standard, which directly affects the performance of airlines and agents. Therefore, we investigate the impact of service level on profits as follows. Firstly, the sensitivity analysis of the service level is carried out to study the change of the system profits $P$ with the change of service level $\alpha$ and the variation trend of the difference value $\Delta \mathrm{P}$ of system profits. The simulation results are listed in Table 6.

Table 6. Sensitivity analysis of service level $\alpha$

\begin{tabular}{l|lllllll}
\hline $\boldsymbol{x}$ & 0.4 & 0.5 & 0.6 & 0.7 & 0.8 & 0.9 & 1 \\
\hline $\mathrm{P}(\mathrm{RMB})$ & 16625 & 19375 & 23125 & 26975 & 27625 & 24375 & 19395 \\
$\Delta \mathrm{P}(\mathrm{RMB})$ & - & 2750 & 3750 & 3850 & 650 & -3250 & -4980 \\
\hline
\end{tabular}

As can be seen from Table 6, as the service level increases, the system profits $P$ and the difference value $\Delta P$ increase first and then decrease: (1) When the service level $\alpha$ is lower than 0.8 , with the increase of $\alpha, P$ shows a trend of quick increase. It is worth noting that the growth rate increases in the early stage while begins to decrease when $\alpha$ is between 0.7 and 0.8 , which confirms the law of diminishing marginal benefits. The marginal utility at this time is still positive, thus, $P$ continues to increase but the growth rate gradually decreases. (2) When $\alpha$ is between $0.8-1, P$ begins to show a downward trend, and the decline rate increases. This is because the marginal utility at this time is reduced to 0 or even negative.

Therefore, we can conclude that as the service level increases, the system profits begin to rise, but the upward trend tends to increase and then decrease because the increase of service level has different impact on revenue and cost. In the early stage, the growth rate of the revenue is larger than that of cost, so the system profits show an increasing trend. However, in the later period, the growth rate of the cost exceeds that of revenue, so the difference between revenue and cost is getting smaller and smaller, and even negative.

In order to provide more targeted measures for airlines and agents, we also examine the impact of service level on profits of airlines and agents, as shown in Fig. 2. 


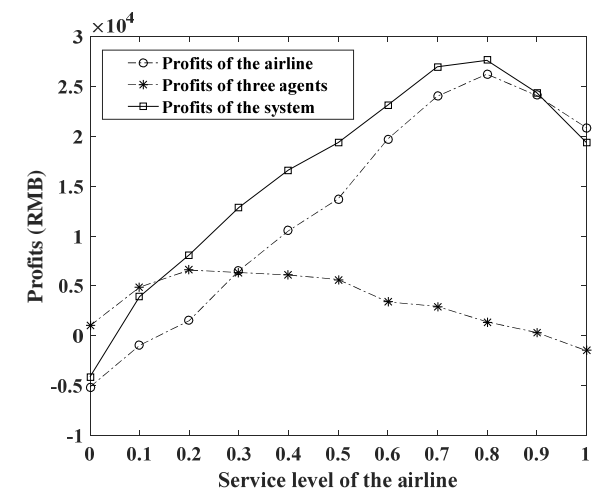

Figure 2. Variations of profits under different service levels

It can be seen from Fig. 2 that the profits of the airline have the similar evolution trend with the system total profits. However, the agents' profits only increased slightly in the early stage, and then it shows a downward trend. This is because the improvement of service level of the airline increases the passenger's trust in civil aviation which benefits agents at first, but this improvement will inevitably lead to a decline in the loyalty of passengers to agents, which will further weaken competitiveness and profits of agents.

Therefore, for the airline, when the service level is lower than 0.8 , the airline should improve the service level, utilize multiple Internet to provide more seats through direct channels, increase the direct channel ratio, design value-added and personalized products by official websites, such as seat reservation, on-board meal pre-selection, taxi reservation, hotel reservations and other personalized services. When the service level is larger than 0.8 , the airline should reduce service costs and channel coordination costs to ensure maximum profits.

The improvement of airline's service level has two impacts on agents. Firstly, passengers are less loyal to agents than airlines, so agents should improve the service level by providing more personalized service than airlines, but the expenses will increase. Secondly, due to the advantages of lower barrier to enter, lower investment costs and risks, agents can obtain large profits to offset the above costs based on airlines' online channels.

\section{The Impact of Service Level on the System Robustness}

The relationship between service level and the system robustness is investigated by observing the change of system robustness with service level. The simulation results are shown in Fig. 3.

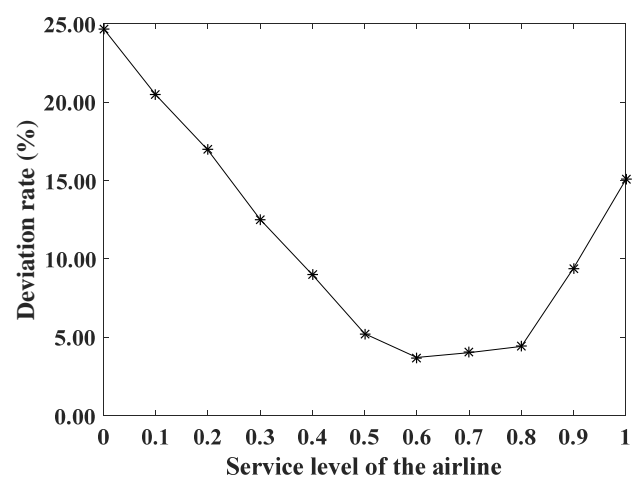

Figure 3. Variations of deviation rate under different service levels

As shown in Fig.3, with the increase of the airline's service level, the system deviation rate shows two trends: (1) When the service level is between 0 and 0.6, the deviation rate shows a downward trend, which means the robustness of the coordination system increases significantly. This is because the improvement of the service level has made the civil aviation market active so that the airline and agents have higher efficiency of channel coordination. (2) When the service level is between 0.6-1, the deviation rate begins to show an upward trend, that is, the higher service level is, the weaker the 
channel coordination system's robustness will be. In this case, the airline and agents have conflicts of interest so the channel coordination efficiency begins to decrease and the robustness declines.

As the service level increases, the system robustness and profits increase first and then decrease. Therefore, the airline should moderately improve the service level. When the service level is raised to 0.6, the service level should not be excessively pursued, and the trade-off between profits and robustness is required. When the service level reaches 0.8 , the service level needs to be controlled because profits and robustness begin to show a downward trend.

\section{Conclusion}

Service level is a key factor of airlines' hybrid distribution channel coordination. Firstly, this paper introduced the model of airlines' hybrid channel coordination with service level; then took numerical experiments of distribution channel ratio and service level and investigated the impact of variation of service level. The paper draws the following conclusions: (1) airlines should pay attention to the impact of service level on themselves, improve service to a moderate level and maintain the optimal service level, which can improve the robustness of the system and increase their own revenue; (2) agents should seize the opportunity to reduce channel costs, provide more personalized user experience than airlines, and carry out cooperation to improve service level to grasp more market demands; (3) airlines and agents should also cooperate with each other, which will help the airlines ' hybrid channel coordination system to be more robust and orderly, thus achieving a win-win situation.

However, as passengers' demands for services are growing higher and higher, and their needs are becoming more diverse, how to quantify the service level is a problem worthy of attention in the future.

\section{Acknowledgement}

This work was supported by the Social Science Foundation of Jiangsu Province (grant number 15GLC001) and the Postgraduate Innovation Base (Laboratory) Open Fund Project of Nanjing University of Aeronautics and Astronautics (grant number kfjj20180706).

\section{References}

[1] C. Jiang, R. Hu, D.Y. Li Research on influence factors of uncertainty in airlines' hybrid channels coordination [J]. Journal of Wuhan University of Technology (Information and management engineering), 2016, 38(2):173-177.

[2] K. Cheng, Z.H. Lee, H. Shomali. Airline firm boundary and ticket distribution in electronic markets[J]. International Journal of Production Economics, 2012, 137(1):137-144.

[3] L. Zhang, X.D. Ding, Z.F. Sun. A game model of airline tickets marketing channel[J]. Mathematics in Practice and Theory, 2014, 44(19):155-161.

[4] S. Saha. Channel characteristics and coordination in three-echelon dual-channel supply chain[J]. International Journal of Systems Science, 2016, 47(3):740-754.

[5] A. David, E. Adida. Competition and Coordination in a Two-Channel Supply Chain[J]. Production \& Operations Management, 2015, 24(8):1358-1370.

[6] C. Jiang, R. Hu, D.Y.Li. Research on robust optimization in airlines' hybrid channels coordination with uncertain demands[J]. Journal of Wuhan University of Technology (Transportation Science \& Engineering), 2017, 41(1):92-96.

[7] C. Jiang, R. Hu, D.Y. Li. Airlines hybrid channels coordination based on fuzzy programming[J]. Aeronautical Computing Technique, 2016, 46(6):64-68. 
[8] S.X. Xie, Q.Z. Xue, R. Li. An approach to customer loyalty [J]. China Civil Aviation, 2002, 22(10):79-83.

[9] R. Yu. Study on improvement of air service quality through experience [J]. Journal of China Civil Aviation Flight University of China, 2011, 22(3):39-41.

[10] X.J. Ma. Research on problems of civil aircraft health management system oriented to custom service [D]. Nanjing University of Aeronautics and Astronautics, 2014.

[11] J.L. Zhao. Improving air service quality pursuant to Zeihtaml and Berry’s service model[J]. Journal of China Civil Aviation University of China, 2000, 18(2):14-17.

[12] J.B Ni. The design and accomplishment of airlines' operating control center management System [D]. Jilin University, 2016.

[13] R. Hu, F.F Zhang. Research on robust optimization in airlines' hybrid channels coordination with random demands[C]. 2018 7th International Conference on Industrial Technology and Management, 2018:298-303.

[14] C.Y.Dye, T.P. Hsieh, L.Y Ouyang. Determining optimal selling price and lot size with a varying rate of deterioration and exponential partial backlogging[J], European Journal of Operational Research, 2007, 181:668-678. 